\title{
Papers
}

\section{Deaths from chickenpox in England and Wales 1995-7: analysis of routine mortality data}

Helen Rawson, Amelia Crampin, Norman Noah

\begin{abstract}
Objective To evaluate the epidemiology and impact of mortality from chickenpox in England and Wales. Design Review of death certificates from the Office for National Statistics on which codes for "chickenpox" or "varicella" were mentioned. Further information ascertained from certifying physician. Participants Those certified as having died from chickenpox in England and Wales, 1995-7.

Main outcome measures Diagnosis and age and sex distributions of deaths from chickenpox.

Results On average, 25 people a year die from chickenpox. Overall case fatality was 9.22 per 100000 consultations for chickenpox. Adults accounted for $81 \%$ of deaths and $19 \%$ of consultations. Deaths were twice as common in men as in women. More of those who died were born outside United Kingdom than expected (12\% v 4\%).

Conclusions Chickenpox is not a mild disease. Deaths in adults are increasing, both in number and proportion.

\section{Introduction}

The age distribution of people with chickenpox and mortality from the disease in developed countries are changing. ${ }^{1}$ In England and Wales deaths in adults have increased in number. ${ }^{2}$ In the United States chickenpox is the leading cause of deaths that could be prevented by vaccine. ${ }^{3}$ As part of a larger study on the burden of chickenpox we reviewed deaths from chickenpox in England and Wales for 1995-7.
\end{abstract}

\section{Methods}

We obtained copies of death certificates that mentioned "chickenpox" or "varicella" (ICD-9 (international classification of diseases, 9th revision) code 052; ICD-10 (10th revision) code B01) for 1995-7 and data on the annual numbers of deaths for 1985-97 from the Office for National Statistics. We sent questionnaires to the physicians responsible for the patients to clarify the diagnosis. Using this information we classified deaths into those in which primary infection with varicella zoster virus was the definite or probable underlying cause of death and those in which it could be excluded. We also reviewed confidential inquiries into maternal deaths in the United Kingdom for 1985-96. ${ }^{4}$ The research unit of the Royal College of General Practitioners provided data on age and sex specific incidences of chickenpox.

\section{Results}

Response rates and varicella classification

We obtained 119 death certificates. Of these, we classified $71(60 \%)$ deaths as definitely or probably due to chickenpox (for example, chickenpox pneumonia, generalised chickenpox, etc). Of these, 58 replies from physicians confirmed $56(97 \%)$ as definite, so we estimated $97 \%$ (12.6) of the 13 remaining cases for which we had no confirmation as definite (table 1). Of the $48(40 \%)$ that we classified as uncertain or not due to chickenpox, 19 of 36 physicians (53\%) confirmed the diagnosis on the certificate. We therefore estimated $53 \%$ (6.3) of the 12 remaining as chickenpox. Thus, of the original 119 deaths, we estimated that $94(79 \%)$ were really attributable to chickenpox (table 1). This is a lower bound value. We had no further information for 25 cases (no reply from physician in charge in 14 cases and notes lost in 11 cases). The overall response rate therefore was $88 \%(105 / 119)$.

In these 94 cases, physicians gave chickenpox as a definite or contributory cause of death in $75(80 \%)$ (table 1). In the 19 remaining cases $(20 \%)$ the disease present was herpes zoster (shingles). Of these 75, nine (12\%, 95\% confidence interval $5 \%$ to $19 \%$ ), all adults, were born outside Europe or North America. Twenty eight patients (37\%) had a known exposure to chickenpox or herpes zoster within the three weeks before onset; 13 (17\%) had no known exposure; and 33 (44\%) were unaware of any exposure. One patient gave no details. From 1985 to 1996 deaths of seven women

Table 1 Classification of death certifications from chickenpox: 1995-7

\begin{tabular}{|c|c|c|c|}
\hline \multirow[b]{2}{*}{ Our classification } & \multicolumn{2}{|c|}{ Physician's classification } & \multirow[b]{2}{*}{ Total } \\
\hline & Chickenpox & Not chickenpox & \\
\hline \multicolumn{4}{|c|}{ Definite or probable chickenpox $(n=71)$} \\
\hline Response from physician & 56 & 2 & 58 \\
\hline No response or notes lost & $12.6^{\star}$ & $0.4^{*}$ & 13 \\
\hline \multicolumn{4}{|c|}{ Uncertain or not chickenpox $(\mathrm{n}=48)$} \\
\hline Response from physician & 19 & 17 & 36 \\
\hline No response or notes lost & $6.3^{*}$ & $5.7^{\star}$ & 12 \\
\hline Total & 94 & 25 & 119 \\
\hline
\end{tabular}

*Estimates.

\author{
Guy's, King's \\ College, and \\ St Thomas's \\ Hospitals School of \\ Medicine and \\ Dentistry, London \\ SE1 8AW \\ Helen Rawson \\ research assistant \\ London School of \\ Hygiene and \\ Tropical Medicine, \\ London \\ WC1E 7HT \\ Amelia Crampin \\ lecturer \\ Norman Noah \\ professor \\ Correspondence to: \\ N Noah \\ norman.noah@ \\ lshtm.ac.uk
}

BMJ 2001;323:1091-3 
Table 2 Estimated case fatality by age group per 100 000, 1995-7

\begin{tabular}{|c|c|c|c|c|c|c|}
\hline & $0-4$ years & $5-14$ years & $15-44$ years & $45-64$ years & $\geqslant 65$ years & Total \\
\hline Deaths (confirmed) & 12 & 2 & 29 & 11 & 21 & 75 \\
\hline No of cases* & 456444 & 211930 & 144597 & 11056 & 2854 & 826881 \\
\hline Rate per $100000(95 \% \mathrm{Cl})$ & 2.63 (1.36 to 4.60$)$ & 0.94 (0.11 to 3.41$)$ & 20.06 (13.43 to 28.80$)$ & 99.49 (49.67 to 178.02$)$ & 735.8 (455.5 to 1124.8 ) & 9.07 (7.13 to 11.37$)$ \\
\hline
\end{tabular}

${ }^{*}$ Cases based on consultation rates for 1995-7, Royal College of General Practitioners.

Table 3 Confirmed deaths from chickenpox by age group and sex, 1995-7. Figures are number (percentage) of deaths*

\begin{tabular}{lccc}
$\begin{array}{l}\text { Age group } \\
\text { (years) }\end{array}$ & Male & Female & Total \\
\hline $0-4$ & $8(10.7)$ & $4(5.3)$ & $12(16.0)$ \\
\hline $5-14$ & $1(1.3)$ & $1(1.3)$ & $2(2.7)$ \\
\hline $15-44$ & $21(28.0)$ & $8(10.7)$ & $29(38.7)$ \\
\hline $45-64$ & $7(9.3)$ & $4(5.3)$ & $11(14.7)$ \\
\hline$\geqslant 65$ & $13(17.3)$ & $8(10.7)$ & $21(28.0)$ \\
\hline Total† & $50(66.7)$ & $25(33.3)$ & $75(100)$ \\
\hline
\end{tabular}

${ }^{*}$ Cases based on consultation rates for 1995-7, Royal College of General Practitioners.

†Fisher's exact test, $\mathrm{P}=0.883$ for difference.

with chickenpox in the third trimester of pregnancy and one in the second trimester were reported to the confidential inquiry. ${ }^{4}$

\section{Estimating case fatality}

We used general practitioners' consultation rates to estimate the number of cases of clinical chickenpox in the population. The case fatality was $9.22 / 100000$ for 1985-97. In each year, the number of deaths certified generally closely paralleled the consultation rate for chickenpox.

In $1995-7,81 \%$ of consultations for clinical chickenpox were for children aged 0 -14 years (table 2 ). Of the certified confirmed deaths due to chickenpox, $61(81 \%)$ were in adults, 58 of whom were aged over 25 years. The case fatality, based on age specific consultation rates, showed considerable variation (table 2). Mortality from chickenpox was most likely in those aged 65 years or older and least likely in those aged 5-14 years (table 3 ). The sex ratio of two male deaths to one female death was especially apparent in those aged 15-44 years, although the consultation rates for chickenpox in both sexes were similar.

\section{Discussion}

Mortality from chickenpox is not negligible. During 1995-7, 81 deaths were recorded by the Office for National Statistics. However, we received 119 certificates that mentioned chickenpox or varicella. After detailed inquiries, we estimated that at least 75 were genuine cases of chickenpox. This suggests at least 25 deaths from chickenpox annually. In 1996-7 there were seven certified deaths from whooping cough, mumps, measles, and Haemophilus influenzae type b (Hib) meningitis in England and Wales compared with 67 from chickenpox.

\section{Specificity of diagnosis}

We asked the clinician concerned to review our presumptive diagnosis taken from the certificate. There was a good response and excellent consistency between our assessment and theirs, especially in those we classified as true chickenpox (consistency 97\%). This suggests high specificity. Sensitivity may have been lower because, conversely, some deaths from chicken- pox may not be correctly coded or certificated, ${ }^{5}$ and we may have lost some. Thus our figure of 25 deaths a year from chickenpox is probably an underestimate.

Misclassification may be partly due to difficulty in distinguishing between primary infection and reactivation of latent varicella zoster causing overwhelming infection in an immunocompromised host and partly to the use of terms such as "herpes varicella zoster" or "disseminated varicella zoster." For estimates of clinical chickenpox we used the research unit database of the Royal College of General Practitioners, which is derived from sentinel reporting. For a common, easily diagnosed disease such as chickenpox it is likely to be representative. For milder forms of the disease it may underestimate, and our case fatalities are likely to be overestimates.

\section{Case fatality and age}

The imbalance in the number of deaths from chickenpox between children and adults in England and Wales has been consistent since 1967. Deaths in adults have increased in number and proportion: they accounted for 48\% (88) of all deaths from chickenpox in 1967-77, $64 \%(120)$ in $1978-85,{ }^{6}$ and $81 \%$ (269) in 1986-97. Adults accounted for over $81 \%$ of the confirmed deaths in our study but for only $19 \%$ of the consultations. Chickenpox is also an important cause of death in children aged 0-4 years because more children in that age group catch chickenpox and because a higher proportion of them die from the illness.

The case fatality and number of deaths in males were twice those in females. The group of men aged 15-44 years accounted for almost half the confirmed male deaths and over a quarter of all confirmed deaths from chickenpox. Underlying HIV infection was reported in only one of our cases. Among the people who died from chickenpox 12\% were born outside Europe or North America compared with the 4\% in the general population of the United Kingdom who were born outside Europe and North America, There seems to be a substantial difference in the transmission of chickenpox in tropical and temperate populations. ${ }^{7}$ Chickenpox causes fewer than one maternal death each year, but this is an important group.

\section{Role of vaccine}

A chickenpox vaccine is available, though not yet licensed in the United Kingdom. Our results do not on their own provide sufficient evidence for mass vaccination. We need information not only on the burden of disease at primary and secondary care levels, but also on the effect of the vaccine on herpes zoster. We also need to ensure a high enough uptake so that the disease does not shift towards the older population and a higher mortality.

The good correlation between the number of deaths annually and the general practitioners' base rate of clinical chickenpox supports our view that death certification from chickenpox does indeed reflect the 
What is already known on this topic

Chickenpox can be fatal, especially in immunosuppressed people and adults

The age distribution of cases has been shifting upwards for about 30 years

\section{What this study adds}

About $80 \%$ of deaths certified as due to chickenpox are due to chickenpox

Chickenpox accounts for about 25 deaths annually in England and Wales, more than from measles, mumps, pertussis, and Hib meningitis combined

Mortality in adults has been increasing for at least 30 years and now $80 \%$ of deaths from chickenpox are in adults

Deaths were twice as common in men as in women

infection accurately enough for our study to be valid. This study confirms that chickenpox causes considerable mortality in adults and may be increasing in importance.
We thank the many physicians who responded to our survey, the staff of the Office for National Statistics, London, and the Royal College of General Practitioners Research Unit, Birmingham, for the supply of data, and Dr Richard Hooper for statistical advice.

Contributors: HR obtained, collated, and analysed the data and contributed to early drafts of the paper. AC contributed to the design and analysis, liaised with the certifying physicians, classified the deaths, and wrote the first draft of the paper. NN conceived and initiated the study, contributed to the interpretation of the data, and wrote the final drafts. $\mathrm{NN}$ will act as guarantor.

Funding: Pasteur Merieux MSD for studies of chickenpox morbidity and mortality.

Competing interests: $\mathrm{NN}$ received a grant and funding for a research assistant from Pasteur-Merieux (manufacturers of a chickenpox vaccine) and has been reimbursed in part for attending a conference.

1 Miller E, Vurdien J, Farrington P. Shift in age in chickenpox. Lancet $1993 \cdot 341 \cdot 308-9$

2 Fairley CK, Miller E. Varicella-zoster virus epidemiology-a changing scene? J Infect Dis 1996;174(suppl 3):314-9.

3 Centres for Disease Control and Prevention. Varicella-related death among children-United States, 1997. MMWR Morb Mortal Wkly Rep $1998 ; 47: 365-8$

4 Department of Health. Report on confidential enquiries of maternal deaths in the UK. London: HMSO and Stationery Office, 1991, 1994, 1996, 1998.

5 Maudsley G, Williams EMI. "Inaccuracy" in death certification-where are we now? J Pub Health 1996;18:59-66.

6 Joseph CA, Noah N. Epidemiology of chickenpox in England and Wales, 1967-85. BMJ 1988;296:673-6.

7 Garnett GP, Cox MJ, Bundy DAP, Didier JM, St.Catharine J. The age of infection with varicella-zoster virus in St Lucia, West Indies. Epidemiol Infect 1993;110:361-72.

(Accepted 14 July 2001)

\title{
Reducing violence in severe mental illness: randomised controlled trial of intensive case management compared with standard care
}

\author{
Elizabeth Walsh, Catherine Gilvarry, Chiara Samele, Kate Harvey, Catherine Manley, Peter Tyrer,
} Francis Creed, Robin Murray, Thomas Fahy for the UK700 Group

\author{
Abstract \\ Objectives To establish whether intensive case \\ management reduces violence in patients with \\ psychosis in comparison with standard case \\ management. \\ Design Randomised controlled trial with two year \\ follow up. \\ Setting Four inner city community mental health \\ services. \\ Participants 708 patients with established psychotic \\ illness allocated at random to intervention (353) or \\ control (355) group. \\ Intervention Intensive case management (caseload \\ 10-15 per case manager) for two years compared \\ with standard case management (30-35 per case \\ manager). \\ Main outcome measure Physical assault over two \\ years measured by interviews with patients and case \\ managers and examination of case notes. \\ Results No significant reduction in violence was \\ found in the intensive case management group \\ compared with the control group (22.7\% v 21.9\%, \\ $\mathrm{P}=0.86)$.
}

Conclusions Intensive case management does not reduce the prevalence of violence in psychotic patients in comparison with standard care.

\section{Introduction}

Serious acts of violence committed by people with mental illness are statistically rare events. ${ }^{1}$ Efforts of community services to prevent violence by the small subgroup at risk may be limited by the lack of effectiveness of standard treatment interventions, inadequate attention to clinical factors associated with violencefor example, drug misuse and poor engagement and treatment adherence by patients-and the difficulty of altering risk associated with impoverished and dangerous living environments. ${ }^{23}$ Fragmentation between services compounds the difficulties.

The care programme approach was introduced, partly to address this fragmentation, after several killings by people with severe mental illness were much reported in the media. ${ }^{4}$ The key elements are assessment of need and risk, development of a care plan, nomination of a responsible key worker, and regular review. Case management incorporates these 